\title{
Evaluation of Neuronavigation in Glioma Surgery
}

\author{
Mahmoud M. Ammar, Mohamed Mahmoud, Ahmed Elsaied Abou Kreasha, Abd Elhay Mousa \\ Department of Neurosurgery, Faculty of Medicine, Assiut University, Assiut, Egypt \\ Email: dr.mahmoud.ammar90@gmail.com
}

How to cite this paper: Ammar, M.M., Mahmoud, M., Kreasha, A.E.A. and Mousa, A.E. (2020) Evaluation of Neuronavigation in Glioma Surgery. Open Journal of Modern Neurosurgery, 10, 36-50. https://doi.org/10.4236/ojmn.2020.101005

Received: October 9, 2019

Accepted: November 25, 2019

Published: November 28, 2019

Copyright $\odot 2020$ by author(s) and Scientific Research Publishing Inc. This work is licensed under the Creative Commons Attribution International License (CC BY 4.0).

http://creativecommons.org/licenses/by/4.0/

\section{cc) (7) Open Access}

\begin{abstract}
Background: Glioma is one of the most common intracranial tumors, the treatment for which is surgical resection. Cerebral gliomas in eloquent brain areas are closely associated with important structures. The deep location of such gliomas renders complete resection difficult overlaying structures can be injured, causing hemiplegia, aphasia, coma, and other severe complications. The degree of resection directly influences the prognosis of patients. The application of neuronavigation enables the accurate localization of lesions during surgery, significantly improving microneurosurgery. The accuracy of navigation, however, has typically been compromised by several factors, including operation error, shifting of registration system, and intraoperative brain tissue deformation (brain shift). Aim of Study: The aim of the study is to evaluate and demonstrate the efficacy of neuronavigation in the management of brain gliomas. Patients and Methods: This is a prospective randomized clinical trial study included 35 patients with brain glioma were operated for excision or biopsy taken. Results: Localization of burr hole and craniotomy flap was excellent in 33 patients (94.2\%) and fault in 2 cases (5.7\%), 29 patients $(82.8 \%)$ had near total excision of the lesions, and 6 patients (17.1\%) had burr hole biopsy, intra operative course was smooth in 33 patients (94.2\%) with no intra operative complications, 2 cases (5.7\%) developed intra operative bleeding that was due to liver problems. Reading the post operative imaging shows that 27 patients $(77.1 \%)$ had mild to moderate post operative edema around the site of the lesion, the other eight cases were free of hemorrhage, infarction or edema. Conclusion: We found that neuronavigation is very helpful in our cases in localization of the lesions, decreasing time of surgery, reducing morbidity and mortality and decreasing hospital stay. There are concerns about navigation systems including time consuming, calculation and registration, restriction of space and view inside the operating field, and so on, we didn't find these issues as obstacles in our work, but we found that there are many advantages that can be helpful in the process of operation.
\end{abstract}




\section{Keywords}

Neuronavigation, Brain Gliomas, Burr Hole Surgery, Localization

\section{Introduction}

Although it was once deemed that "the only good craniotomy is a large craniotomy", image-guided stereotactic techniques and, in particular, surgical navigation have led to smaller, strategically placed and sized craniotomies. Minimal access craniotomies may have several advantages, including reduced length of surgery, lower incidence of wound infections, and shorter length of hospital stay. The minimum size of a craniotomy is, in part, dependent on the size and depth of the lesion as well as on surgical instrumentation [1]. For intra parenchymal lesions at the cortical surface, the craniotomy generally should be large enough to encompass the extent of presentation of the tumor on the surface. For deeper lesions, the craniotomy may not need to be as large as if the lesion presented at the surface because the skull opening can be considered the apex of a working cone extending down to the tumor. Of course, the opening must be large enough for the surgical instruments to fit, as well as for proper illumination and visualization of the region of work [2]. Primary glial neoplasms, especially astrocytomas in adults, behave clinically in a malignant fashion because of their infiltrating nature, making them microscopically truly unrespectable given that surgical resection is an important component in the management of patients with intracranial gliomas, neuronavigation can improve orientation within brain tissue at the time of surgery and assist in determining the margins of solid tumor tissue with surrounding infiltrated brain should improve the safety and efficacy of these procedures [3].

Neuronavigation can be used to perform biopsies with minimal trauma to the brain. A lockable trajectory arm is secured to the Mayfield clamp. Two mm drill guide with a registration star attached is then used in conjunction with a concentric ring target view that enables the required trajectory to be determined. The addition of a virtual tip extension to the drill guide confirms that the target is at the centre of the trajectory. The drill guide is then secured in the lockable trajectory arm and a small twist drill hole performed through the guide [4].

\section{The Aim of This Study}

To evaluate and demonstrate the efficacy of neuronavigation in the management of Glioma.

\section{Patients and Methods}

This is a prospective randomizing clinical trial study including 35 patients with different sites glioma, were admitted and operated in the Neurosurgery Department, Assiut University Hospital and el gala military hospital. The results of us- 
ing the neuronavigator were reviewed to study efficacy of neuronavigation in the management of glioma.

\subsection{Selection Criteria}

- Inclusion criteria: all patients with different sites glioma, inaccessible for resection and the only needed are biopsy.

- Exclusion criteria: patients unfit for surgery, old patients more than $80 \mathrm{yrs}$, infants due to fragile skull not withstanding Mayfield.

\subsection{Preoperative Preparation}

All patients underwent general and neurological assessment including a thorough history, physical examination, laboratory work-up and special study, i.e. neuro-ophthalmological and radiological studies including MRI and CT with and without contrast with navigation protocol.

\subsection{Operative Management}

1) Neuronavigation System

Intraoperative image guidance was achieved by Brain Lab Vectrovision (Munich, Germany) which consists of a mounted array of three charge-coupled device, cameras, a mobile computer workstation with a high resolution monitor, a dynamic reference frame, attached to the Mayfield head holder and a free handheld stereotactic pointing device.

2) Neuronavigation Technique

After induction of anesthesia; the patient was positioned on the table with the patient's head fixed using the Mayfield head clamp, the dynamic reference arc was attached to the Mayfield head clamp and positioning of the camera array was done. Using the reference probe, registration was then carried out; at the end of registration the exact centre of each marker in the images was defined and stored on the workstation screen. Once registered, the dynamic position of the pointer relative to the patient's head was displayed superimposed, upon the images on the screen and the surgeon can further check the level of accuracy by placing the pointer tip on visible structures (such as the nose, ear, ...) and correlating the position of the pointer upon the screen Once the patient was registered with the 3-D image at a specified accuracy level, surgery was performed Using a hand-held pointer, craniotomy site or burr hole was planed according to the exact lesion margins. The incision site was marked; the intra-operative positions were checked periodically using the reference probe whenever necessary.

3) Operative Technique

Patients were positioned with slight flexion of the knee. The head was elevated above the heart 15 degree to help venous drainage and eyes were protected. The planned craniotomy was elevated and lesion was resected or biopsy was taken using standard technique. The wound was closed in layers, For each case, the length of scalp incision, size of craniotomy was assessed, the accuracy of the 
bone flap or burr hole localization was assessed in relation to the lesion and the extent of tumor removal whether (totally or sub totally or biopsy), was reported, The volume of blood loss was estimated. Any complications that occurred during surgery were reported.

\subsection{Post Operative}

All patients were assessed clinically and radiologically in early postoperative period during hospital stay to detect any complications.

\section{Results}

- This study include 35 patients, 15 males (42.8\%) and 20 females (57.1\%) with age groups ranged from (30-60 yrs) as shown in Table 1, the presenting symptoms were headache in 15 patients $(42.2 \%)$, the headache in those patients was typical of increased intra cranial pressure, 5 patients (14.2\%) presented with weakness in the form of hemi paresis, 10 patients (28.5\%) presented with epileptic seizures as shown in Table 2.

- 25 patients $(71.4 \%)$ where free with no motor or sensory affection, no cranial nerves affection and no papilledema (they were complaining of headache, blurring of vision and epileptic seizures), 5 patients (14.2\%) has hemi paresis of different grades: (three patients have grade 4 and two patients have grade 2). Five patients (14.2\%) have papilledema on fundus examination.

- Reading the preoperative images of the patients including CT and MRI with and without contrast, we found that our patients were diagnosed to have glioma of different sites as shown in Table 3 with the size was ranging from $(2-6) \mathrm{cm}$.

- Localization was excellent in 33 patients (94.2\%) and fault in 2 cases (5.7\%), the average duration of surgery was 2.8 hours as it was one hour in 6 patients (17.1\%) with Burr hole biopsy and was 1 - 3 hours in 29 patients $(82.8 \%)$ with craniotomy.

- 29 patients $(82.8 \%)$ had near totally excision of the lesions, and 6 patients (17.1\%) had burr hole biopsy.

- The intra operative course was smooth in 33 patients (94.2\%) with no intra

Table 1. Number and sex of the patients.

\begin{tabular}{ccc}
\hline sex & No. & $\%$ \\
\hline Male & 15 & 42.8 \\
Female & 20 & 57.1 \\
\hline
\end{tabular}

Table 2. Presenting symptoms.

\begin{tabular}{ccc}
\hline presenting symptom & NO & $\%$ \\
\hline Headache & 15 & 42.2 \\
Weakness & 5 & 14.2 \\
Seizures & 10 & 28.5 \\
\hline
\end{tabular}


operative complications, 2 cases (5.7\%) developed intra operative bleeding that was due to liver problems in one case and high vascularity of the lesion in the other case.

- No significant blood loss in six cases who had burr hole biopsy. Blood loss was recorded in the cases with craniotomy as shown in Table 4.

- Five patients developed new motor deficits after surgery in form of hemiparesis and this could be due to the site of the lesion (that was near motor strip) and surgical manipulation, for all of them dehydrating measures, steroids and neurotonics were given to them, all of them showing improvement with physiotherapy in the follow up.

- Eight patients have the same headache but slighter than that of pre-operative, 2 patients have the same diminution of vision, 4 patients have dysphasia as the site of the lesion was left temporal and 11 patients were free from any post operative complications.

- Reading the post-operative imaging of patients of this study showed that: 27 patients $(77.1 \%)$ had mild to moderate post operative edema around the site of the lesion the other eight cases were free of hemorrhage, infarction or edema.

- Hospital stay: depends on the procedure done and the post operative status of the patient as shown in (Table 5).

Table 3. Different sites of the lesions.

\begin{tabular}{cc}
\hline Site & NO \\
\hline Brain stem & 1 \\
Frontal & 14 \\
Fronto parietal & 1 \\
Intra ventricular & 2 \\
Occipital & 2 \\
Parietal & 5 \\
Temporal & 3 \\
Temporoparietal & 3 \\
Thalamic & 4 \\
\hline
\end{tabular}

Table 4. Amount of blood loss.

\begin{tabular}{cc}
\hline BLOOD LOSS & NO \\
\hline NO blood loss & 6 \\
$200-400$ & 17 \\
$400-800$ & 12 \\
\hline
\end{tabular}

Table 5. Duration of hospital stay.

\begin{tabular}{ccc}
\hline & NO & DAYS \\
\hline Burr hole biopsy & 6 & 1 day after surgery \\
Craniotomy of glioma & 29 & 3 days - 6 days after surgery \\
\hline
\end{tabular}




\section{Illustrative Cases}

Case 1: 40 years old female, presented with RT sided focal seizures On examination:

RT sided weakness grade 4, intact sensation, preserved reflexes.

Lab investigation and brain imaging were done including navigation protocol.

Brain CT and MRI showing left temporoparietal intra axial SOL as shown in Figure 1, Figure 2.

Neuronavigation was used for localization of the lesion and excision was done as shown in Figure 3.

Histopathology report was a: gliomablastoma multi form and imaging post operative was done as shown in Figure 4.

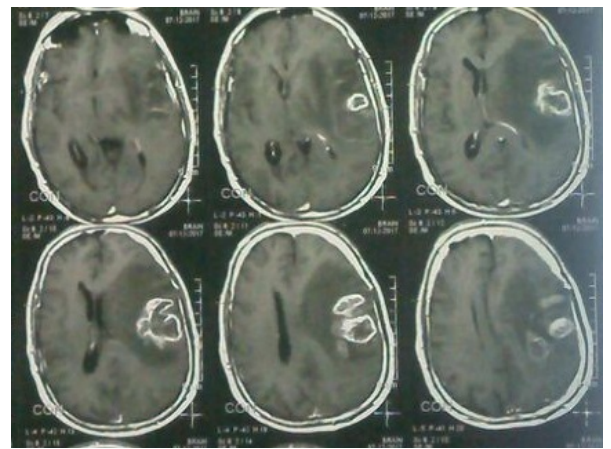

Figure 1. MRI axial cuts showing lt parietal sol.

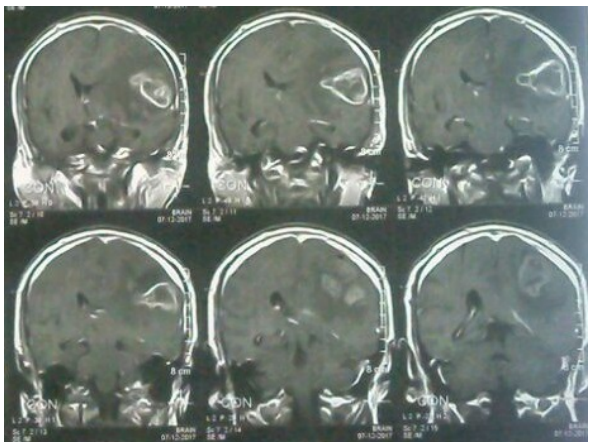

Figure 2. Coronal MRI of lt parietal intra axial sol.

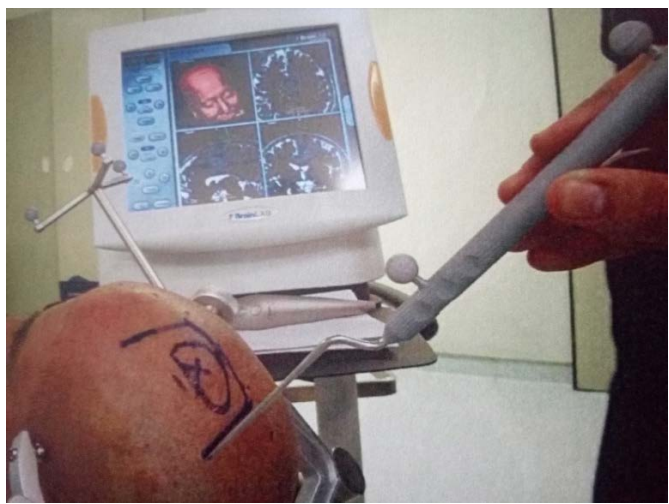

Figure 3. Intra operative localization using navigation. 


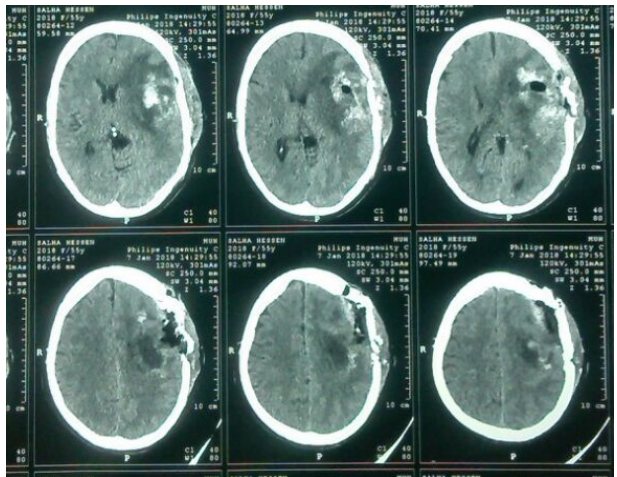

Figure 4. Post operative CT showing near total excision.

Case 2: Female patient 35 years old, presented with severe headache On examination:

She is fully conscious, intact power, sensation and reflexes.

Lab investigation and brain imaging were done including navigation protocol. Brain imaging showed left thalamic lesion as shown in Figure 5 and Figure 6.

Pt was prepared for burr hole biopsy using neuronavigation which help us in direct attack of the lesion with good localization as shown in Figure 7.

Histopathology: report was glioblastoma multiform and ct post operative was done as shown in Figure 8.

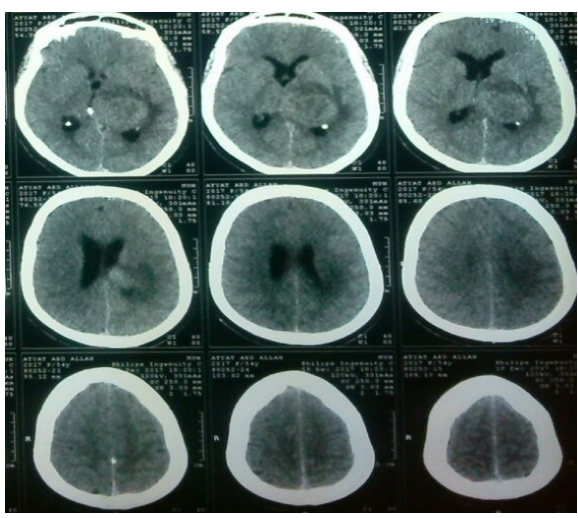

Figure 5. Axial CT preoperative showing lt deep parietal intra axial SOL.

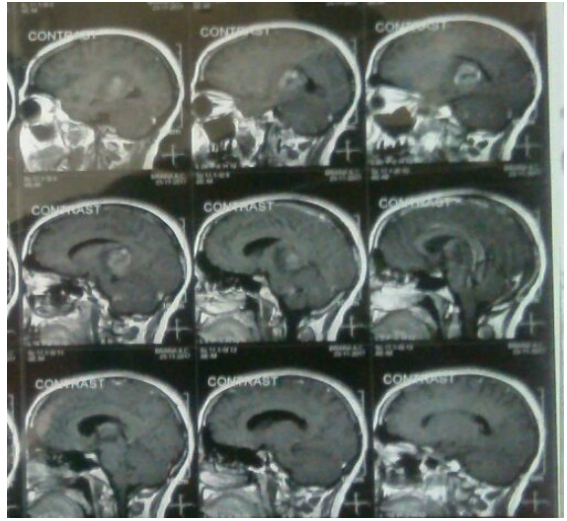

Figure 6. Preoperative sagittal MRI with contrast showing lt deep parietal intra axial SOL. 


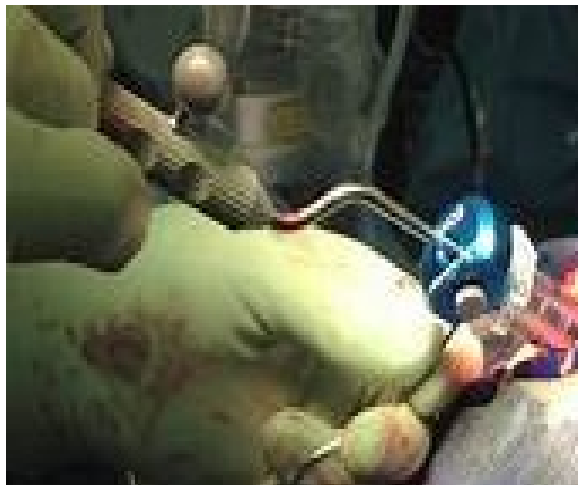

Figure 7. Intraoperative burr hole biopsy using neuronavigation and the prope used to take biopsy.

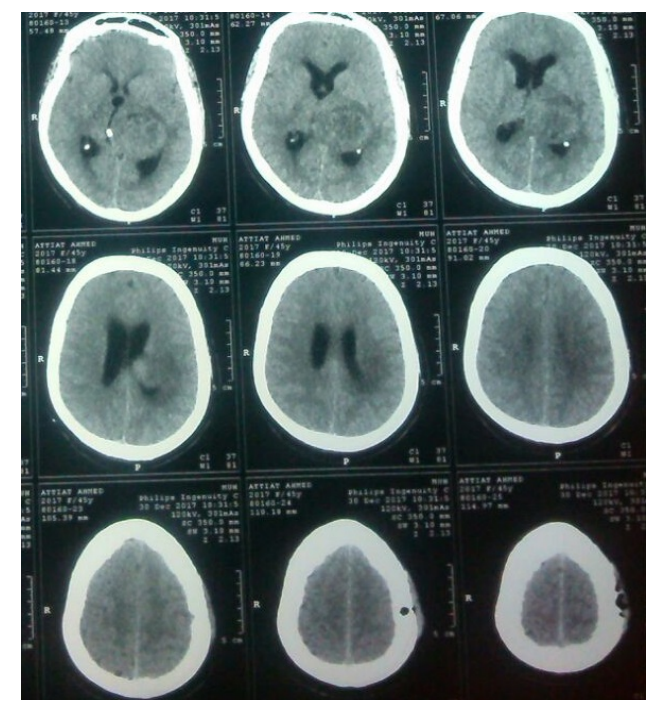

Figure 8. Post operative CT axial cuts after burr hole biopsy.

\section{Discussion}

Neurosurgery currently relies on navigation more than any other specialty due to the particular features of the brain that impose the requirements for perfect orientation, extreme precision, and minimal invasiveness of the neurosurgical procedures [5].

Neuronavigation is a valuable tool for neurosurgeons as it provides them with image-guidance during a surgical procedure that enables surgeons to pinpoint the exact location of the lesion before and during surgery [6].

Glioma resection utilizing neuronavigation might leads to maximization of the extent of resection and so improvement of the survival, and reduction of the incidence of the disabilities, these go with the conclusion of Mikuni \& Miyamoto [7] in evaluation of glioma resection utilizing the neuronavigation.

In this study it was found that, the localization of the lesions was excellent in 33 patients (94.2\%). Naftel et al. [8], Schipper et al. [9] and Tirakotai et al. [10], reported that, neuronavigation provided a high degree of accuracy in lesion targeting, permitting a good anatomical orientation and minimize the brain trauma. 
Zamorano et al. [11] found that this system provided precise lesion localization without limiting the line of vision, the mobility of the surgeon, or the flexibility of instruments. Al-abyad [12] reported that, the neuronavigation was found to be very helpful in preoperative planning for lesions lying near central sulcus or other critical areas. This system has also been shown to provide accurate planning, and resection control images during surgery were obtained with precise localization of residual tumor tissue.

Du et al. [13] reported that, in a 75 cases of brain gliomas, operated upon using neuronavigation, total tumor removal was achieved in 62 of cases $(82.7 \%)$, and subtotal removal was achieved in 13 of cases (17.3\%).

Xue \& Zhang [14] assessed the clinical value of neuronavigation in the resection of intracranial glioma in 54 patients, and they reported that the tumors and the surrounding structures were located accurately. Among the 54 cases, total removal was achieved in 36 cases, (66.6\%) subtotal removal in 10 cases $(18.5 \%)$ and partial removal in 8 cases $(14.8 \%)$.

Jia et al. [15] evaluated the application of neuronavigation in the resection of superficial intracranial tumors in 37 patients, those were compared with a controlled group of 30 patients with tumors in the cerebral hemisphere underwent conventional craniotomy, they found that the tumor localization was accurate and the resection was complete more in the neuronavigation group.

Kurimoto et al. [16] reported that, the percentage of gross total resection was significantly higher in the neuronavigation group compared to that in the no-navigation group (64.3\% vs. $38.2 \%)$.

Michael et al. [17] concluded that neuronavigation was a useful adjunct in the operative management of patients with intracranial lesions as they found that, with neuronavigation tumor resection was complete in all patients as determined with postoperative MRI.

$\mathrm{Xu}$ et al. [18] explored the value of the neuronavigation in surgery of intracranial lesions in 33 patients. The results were; all lesions were targeted precisely. Total removal was achieved in 25 cases (75.7\%). Among them, total removal rate in glioma group ( 9 cases) was $44.4 \%$ (4/9), and total removal rate in other lesions was $87.5 \%(21 / 24)$.

In our study extent of resection was near total excision in 29 cases (82.8\%) as the lesions were diffuse with no definite line of demarcation, burr hole biopsy was done in 6 cases as the lesions were deep, affecting thalamus, or brain stem.

The duration of surgeries was about one hour in burr hole biopsy cases (6 cases), and in craniotomy cases (29 cases) it was 1 - 4 hours. The duration was long in these cases due to some factors including: site, vascularity of the lesion, and preparation of neuronavigation system. Many authors found that there was a slight increase in operation time using navigation system compared to conventional techniques, ranging from 10 - 23 minute [3] [19] [20] [21].

Another study [22] showed that the mean preparation time was $37.8 \mathrm{~min}$ (median $37.5 \mathrm{~min}$ ) without and $68.2 \mathrm{~min}$ (median $72.5 \mathrm{~min}$ ) with neuronavigation $(p<0.001)$. On average neuronavigation added $30.4 \mathrm{~min}$ to the overall 
preparation time compared to standard microsurgical cases. Since no other difference in the preparation procedure could be identified, this increase was attributed to installing and referencing of the navigation systems, including sterile draping of the arm, pointers or reference frames. The mean operative times, on the other hand, were exactly the same for both groups: $288.7 \mathrm{~min}$ (median 275 $\mathrm{min}$ ) without and $288.6 \mathrm{~min}$ (median $260 \mathrm{~min}$ ) with neuronavigation $(p=0.99$ ). The total surgical time taken as the sum of the preparation and operation times was accordingly longer in neuronavigation cases but the difference was not statistically significant $(p=0.11)$.

On the other hand, Schiffbauer et al. [23] reported that mean duration of surgery was 3:05 hrs, for surgery involving neuronavigation, this difference in time may be due to the fact that they used ultra sonography to correct the brain shift which was not used in our study.

When reviewing our cases we found that the intra operative course was smooth in $94.2 \%$ of cases and bleeding occurs in 2 cases, this was actually not due to the use of neuronavigation, but due to the nature of the tumor, which was highly vascular.

The use of neuronavigation was shown to improve the postoperative Quality of life of patients suffering from brain and spinal tumors [24].

In the present series it was found that, the postoperative complications were encountered in the form of hemi paresis in 8 cases, dysphasia in 4 cases and there was no early postoperative mortality.

Paleologos, et al. [25] reported that, the post-operative complications were encountered in $14 \%$ of the cases in the standard surgical group and $6 \%$ of the cases in the neuronavigation group. Similar results was reported by Kurimoto et al. [16] as they found that, postoperatively neurological deterioration occurred in $9.5 \%$ and in $17.6 \%$ of the patients after surgery with neuronavigation and surgery without neuronavigation, respectively.

In our study, hospital stay of the patients was 1 day in burr hole biopsy cases and 3 - 6 days in glioma resection cases.

Germano et al. [26] reported that the neuronavigation is an accurate device that offers clinical benefits; these include precise surgical resection and decreased hospitalization time, as the intra operative extent of resection was accurate in tumor resection in patients undergoing surgery involving the neuronavigation, as confirmed with postoperative images. Also had a significantly shorter hospital stay than those undergoing surgery involving conventional procedures ( $7.5 \pm 1$ versus $10.8 \pm 1.3$ days respectively). On the other hand, Bademci et al. [27] reported that, overall duration of procedures and the duration of hospital stay seemed slightly higher for neuronavigation in comparison to the standard procedures, although it is more effective in the selection of the best position for the surgical approach, reduction of the time required for skin incision, craniotomy planning and cortical incision.

Neuronavigation systems have been developed for the aid of the accurate resection of brain tumors. Therefore, the accuracy of the neuronavigation is im- 
portant. However, many factors can reduce the navigation accuracy during surgery [28].

The brain shift didn't assessed in this study as it need intra operative imaging with intra operative MR or intra operative UIS to update the neuronavigation which are not available in the centers where this study was carried out. But it is important to shy the light on this phenomenon of brain shift as it is the main obstacle against conventional neuronavigation. In fact, there were no major complications or abandoning of image guided system attributed to the effect of brain shift in this study.

Bakay RAE [29] quantified brain shifts during open cranial surgery to assess the impact of post imaging brain distortion on neuronavigation and reported a mean shift of $4.6 \mathrm{~mm}$ of the cortical surface after the Dural opening and $5.7 \mathrm{~mm}$ at the completion of tumor resection.

Hastreiter et al. [30] analyzed the brain shift in 32 gliomas in order to evaluate the maximum displacement of the brain surface and the deep tumor margin. They observed a great variability of the brain shift ranged up to $24 \mathrm{~mm}$ for cortical displacement and exceeding $3 \mathrm{~mm}$ for the deep tumor margin in $66 \%$ of all cases.

Witteka et al. [31] reported that following tumor resection, the brain surface sank $7 \mathrm{~mm}$ below the dural level in $60 \%$ to $70 \%$ of patients in whom it was assessed. This surface brain shift may interfere with the identification of eloquent cortical areas during neuronavigation.

Xu et al. [18] investigated the strategy of brain and lesion shift in 20 patients with supra tentorial cerebral lesions and reported that; for cerebral lesions with clear boundaries, the aim of applying neuronavigation is to find lesions directly and reduce brain injury. For lesions without clear boundaries, neuronavigation is turned to figure out the imaging enhanced border as a guide for complete removal.

Neuronavigation can be updated with intra operative image data without repeated patient registration, facilitating the update procedure. Updated navigation allows achieving enlarged resections and compensates for the effects of brain shift [32]. However, because of the heterogeneous nature of lesions neurosurgeons have to remove, the modest quantity of shared common variance, and the differences between superficial and sub cortical brain shift, it seems unlikely that the amount and course of brain shift become exactly predictable preoperatively. But only an intra operative update of image data should have the capacity to overcome this fundamental problem of modern neuronavigation [33].

Definition of the eloquent areas by the functional MRI and Magneto encephalography (MEG) and incorporation of this information in the image data for neuronavigation can be helpful for planning a trajectory that avoided these regions and minimized the morbidity of the procedure [34].

The advantages of fMRI are the wide availability of the MRI facilities, absence of ionizing radiation and the provision of both the anatomical and functional 
data utilizing the same imaging modality by image fusion with a three dimensional MRI [35].

In the present study there were cases that developed new neurological deficit (8 cases) in the form of hemi paresis; this might be due to affection of motor cortex during manipulation of the tumor which can be avoided by functional imaging.

In general, it has been observed that craniotomies under neuronavigation are smaller and more precise owing to the accuracy afforded by image guidance which leads to; time-saving, reduction of blood loss, confidence to pinpoint lesions below the cortical surface and minimize access corridor and trauma which are regarded as a significant advance [36].

It is the belief that, the widespread adoption of neuronavigational technology will lead to enhanced accuracy of surgery, minimal access to the lesion and minimized trauma, improved operative planning and anatomical appreciation, better intra operative orientation and markedly enhanced surgical confidence. It is anticipated that, as a result of these points and as part of general progress in surgical science, there will be increase patient's safety and better outcomes [37].

To make neuronavigation truly effective, an intraoperative imaging system is required which will further push up the cost [38].

Neuronavigation have its advantages like increased perception of safety, probably increases the amount of tumor resection, along with improved accuracy which may lead to lesser complications. However, the high expense, absence of intra operative imaging, lack of trained dedicated supporting technical staff does make the neuronavigation a strain on the resources [39].

\section{Conclusions}

- We found that neuronavigation is very helpful in our cases in localization of the lesions, decreasing time of surgery, reducing morbidity and mortality of the cases and decreasing hospital stay leading to save of the resources.

- There are concerns about navigation systems including time consuming, calculation and registration, restriction of space and view inside the operating field; and so on we didn't find these issues as obstacles in our work.

- Although neuronavigation provides numerous advantages to the neurosurgeons, it does not in its self improve surgical skills and it remains important that the surgeons should possess a profound anatomical knowledge and a good neurosurgical education.

- Neuronavigation in brain tumor surgery is still in its infancy, and many neuronavigator systems are just developing into their second generation. New possibilities will open up in the forthcoming years leading neurosurgery to completely new horizons in the treatment of brain tumor patients.

\section{Conflicts of Interest}

The authors declare no conflicts of interest regarding the publication of this paper. 


\section{References}

[1] Bianco Ade, M., Miura, F.K., Clara, C., et al. (2013) Low-Grade Astrocytoma: Surgical Outcomes in Eloquent versus Non-Eloquent Brain Areas. Arquivos de Neuro-Psiquiatria, 71, 31-34. https://doi.org/10.1590/S0004-282X2012005000017

[2] Barnett, G.H. (2011) Youmans Neurological Surgery 6th Edition Navigation for Brain Tumors. Elsevier, Philadelphia, Volume 2, Ch. 118, 1293-1299. https://doi.org/10.1016/B978-1-4160-5316-3.00121-0

[3] Yang, S., Law, M., Zagzag, D., et al. (2003) Dynamic Contrast-Enhanced Perfusion MR Imaging Measurements of Endothelial Permeability: Differentiation between Atypical and Atypical Meningiomas. American Journal of Neuroradiology, 24, 1554-1559.

[4] Garslaundt, O., Behari, S., Gralla, J., Fahlbush, R. and Nimsky, C. (2002) Neuro-Navigation: Concept, Techniques and Applications. Neurology India, 50, 244-255.

[5] Keles, G.E. and Berger, M.S. (2004) Advances in Neurosurgical Technique in the Current Management of Brain Tumors. Seminars in Oncology, 31, 659-665. https://doi.org/10.1053/j.seminoncol.2004.07.008

[6] Koivukangas, J. and Pirila, T. (2008) Computer Guided Surgery for Neurosurgery. Annales Chirurgiae et Gynaecologiae, 79, 192-196.

[7] Mikuni, N. and Miyamoto, S. (2010) Surgical Treatment for Glioma: Extent of Resection Applying Functional Neurosurgery. Neurologia Medico-Chirurgica, 50, 720-726. https://doi.org/10.2176/nmc.50.720

[8] Naftel, R.P., Tubbs, R.S., Reed, G.T. and Wellons, J.C. (2010) Planning of Surgical Approach to Intracerebral Tumors of Hemispheres Using Neuronavigation. $Z H$ VOPR NEIROKHIR IM N N BURDENKO Journal, No. 2, 9-13.

[9] Schipper, J., Maier, W., Arapakis, I., et al. (2004) Navigation as a Tool to Visualize Bone-Covered Hidden Structures in Transfrontal Approaches. The Journal of Laryngology \& Otology, 118, 849-856. https://doi.org/10.1258/0022215042703651

[10] Tirakotai, W., Riegel, T., Sure, U., Bozinov, O., et al. (2004) Clinical Application of Neuronavigation in a Series of Single Burr Hole Procedures. Acta Neurochirurgica, 65, 57-64. https://doi.org/10.1055/s-2004-816237

[11] Zamorano, L., Jiang, Z. and Kadi, A.M. (1994) Computer Assisted Neurosurgery System: Wayne State University Hardware and Software Configuration. Computerized Medical Imaging and Graphics, 18, 257-271. https://doi.org/10.1016/0895-6111(94)90050-7

[12] AL-abyad, A.G. (2007) Image-Guided versus Free Hand Preoperative Planning of Craniotomy for Superficial Brain Lesions. EJNS, 22, 53-65.

[13] Du, et al. (2003) ALA-Glioma Study Group Extent of Resection and Survival in Glioblastoma Multiforme: Identification of an Adjustment for Bias. Neurosurgery, 62, 564-576. https://doi.org/10.1227/01.neu.0000317304.31579.17

[14] Xue, X. and Zhang, J. (2008) China Experience in Surgery of Intracranial Astrocytic Tumors Assisted by Electromagnetic Neuronavigation: Report of 54 Cases. Chinese Journal of Neuro-Oncology, 5, 114-116.

[15] Jia, D.Z., Jiang, Y.Q., Li, X.G., et al. (2006) Application of Neuronavigation in the Resection of Superficial Intracranial Tumors with Small Incision. Chinese Journal of Minimally Invasive Neurosurgery, 2006-05-010.

[16] Kurimoto, M., Hayashi, N., Kamiyama, H., Nagai, S., et al. (2004) Impact of Neu- 
ronavigation and Image Guided Extensive Resection for Adult Patients with Supratentorial Malignant Astrocytomas: A Single Institution Retrospective Study. Minimally Invasive Neurosurgery, 47, 278-283. https://doi.org/10.1055/s-2004-830093

[17] Lawton, M.T., Golfinos, J.G., et al. (1998) The State of the Art of Neuronavigation with Frameless Stereotaxy in Intracranial Neurosurgery. Operative Techniques in Neurosurgery, 1, 27-38. https://doi.org/10.1016/S1092-440X(98)80006-6

[18] Xu, X.B., Zhang, J.L., Li, G., et al. (2006) Neuronavigated Microsurgical Resection of Intracranial Lesions. Chinese Journal of Neurosurgical Research, 78, 11-12.

[19] Perlick, et al. (2004) Volumetric Stereotactic Surgical Resection of Intraxial Brain Mass Lesions. Mayo Clinic Proceedings, 63, 1186-1198. https://doi.org/10.1016/S0025-6196(12)65405-6

[20] Grutzner, et al. (2004) Computer Guided Surgery for Neurosurgery. Annales Chirurgiae et Gynaecologiae, 79, 192-196.

[21] Mielke, et al. (2001) Emprical Studies of the Reliability of Surgical Navigation Systems. Biomedizinische Technik, 47, 37-40.

[22] Karataç, A., et al. (2005) Some Collected Principles of Microneurosurgery: Simple and Fast, While Preserving Normal Anatomy: A Review. Surgical Neurology, 64, 195-200. https://doi.org/10.1016/j.surneu.2005.04.031

[23] Schiffbauer, H., Berger, M.S., Ferrari, P., Freudenstein, D., Rowley, H.A. and Roberts, T.P. (2002) Neuronavigation in Brain Tumor Surgery. Journal of Neurosurgery, 97, 1333-1342. https://doi.org/10.3171/jns.2002.97.6.1333

[24] Haberland, et al. (2000) Virtual Reality System for Planning Minimally Invasive Neurosurgery. Technical Note. Journal of Neurosurgery, 108, 382-394. https://doi.org/10.3171/JNS/2008/108/2/0382

[25] Paleologos, T.S., Wadley, J.P., Kitchen, N.D. and Thomas, D.G. (2000) Clinical Utility and Cost Effectiveness of Interactive Image Guided Craniotomy: Clinical Comparison between Conventional and Image Guided Meningioma Surgery. Neurosurgery, 47, 40-48. https://doi.org/10.1227/00006123-200007000-00010

[26] Germano, I.M., Villalobos, H., Silvers, A. and Post, K.D. (2009) Clinical Use of Optical Digitizer for Intracranial Neuronavigation. Neurosurgery, 45, 261-269. https://doi.org/10.1097/00006123-199908000-00013

[27] Bademci, G. (2007) Clinical Experiences in Neuronavigation. Turkiye Klinikleri Journal of Medical Sciences, 27, 195-200.

[28] Dwarakanath, et al. (2007) Assessment of Image Guided Accuracy in a Skull Model: Comparison of Frameless Stereotaxy Techniques vs. Frame-Based Localization. Journal of Neuro-Oncology, 76, 65-70. https://doi.org/10.1007/s11060-005-2915-z

[29] Bakay, R.A.E. (1998) Intraoperative Brain Shift and Deformation: A Quantitative Analysis of Cortical Displacement in 28 Cases. Neurosurgery, 43, 759-760. https://doi.org/10.1097/00006123-199810000-00013

[30] Hastreiter, P., Rezk-Salama, C., Soza, G., Bauer, M., et al. (2004) Strategies for Brain Shift Evaluation. Medical Image Analysis, 8, 447-464. https://doi.org/10.1016/j.media.2004.02.001

[31] Witteka, K.R., Warfield, S.K. and Miller, K. (2005) Brain Shift Computation Using a Fully Nonlinear Biomechanical Model. Medical Image Computing and Computer-Assisted Intervention, 8, 583-590. https://doi.org/10.1007/11566489_72

[32] Lunsford, L.D., Niranjan, A., Kassam, A., Khan, A., Amin, D. and Kondziolka, D. (2008) Intraoperative Image: Evaluations, Options and Practical Applications. Clinical Neurosurgery, 55, 76-86. https://doi.org/10.1159/000147636 
[33] Letterboer, M.M.J., Willems, P.W.A., Viergever, M.A. and Niessen, W.J. (2005) Brain Shift Estimation in Image Guided Neurosurgery Using 3-D Ultrasound. Neurosurgery, 52, 268-276. https://doi.org/10.1109/TBME.2004.840186

[34] Rutten, G.J., Ramsey, N., Noordmans, H.J., Willems, P., et al. (2003) Toward Functional Neuronavigation: Implementation of Functional Magnetic Resonance Imaging Data in a Surgical Guidance System. Neurosurgical Focus, 15, E6. https://doi.org/10.3171/foc.2003.15.1.6

[35] Nimsky, C., Ganslandt, O. and Fahlbusch, R. (2004) Functional Neuronavigation and Intraoperative MRI. Advances and Technical Standards in Neurosurgery, 29, 229-263. https://doi.org/10.1007/978-3-7091-0558-0_6

[36] Upadhyay, U.M. and Golby, A.J. (2008) Role of Pre and Intraoperative Imaging and Neuronavigatin in Neurosurgery. Review of Medical Devices, 5, 65-73.

https://doi.org/10.1586/17434440.5.1.65

[37] Roberts, D.W., Strohbehn, J.W., Hatch, J.F., et al. (1986) A Frameless Stereotaxic Integration of Computerized Tomographic Imaging and the Operating Microscope. Journal of Neurosurgery, 65, 545-549. https://doi.org/10.3171/jns.1986.65.4.0545

[38] Hall, W.A. and Truwit, C.L. (2008) Intraoperative MR-Guided Neurosurgery. Journal of Magnetic Resonance Imaging, 27, 368-375. https://doi.org/10.1002/jmri.21273

[39] Jenny and Boeri (2002) Neuronavigation Assisted Meningioma Surgery. Medical Journal, 9, 3-5. 